\title{
Pattern recognition of star constellations for spacecraft applications
}

\author{
Liebe, Carl Christian
}

Published in:

I E E E Aerospace and Electronic Systems Magazine

Link to article, DOI:

$10.1109 / 62.180383$

Publication date:

1993

Document Version

Publisher's PDF, also known as Version of record

Link back to DTU Orbit

Citation (APA):

Liebe, C. C. (1993). Pattern recognition of star constellations for spacecraft applications. I E E E Aerospace and Electronic Systems Magazine, 8(1), 31-39. https://doi.org/10.1109/62.180383

\section{General rights}

Copyright and moral rights for the publications made accessible in the public portal are retained by the authors and/or other copyright owners and it is a condition of accessing publications that users recognise and abide by the legal requirements associated with these rights.

- Users may download and print one copy of any publication from the public portal for the purpose of private study or research.

- You may not further distribute the material or use it for any profit-making activity or commercial gain

- You may freely distribute the URL identifying the publication in the public portal

If you believe that this document breaches copyright please contact us providing details, and we will remove access to the work immediately and investigate your claim. 


\title{
Pattern Recognition of Star Constellations for Spacecraft Applications
}

\author{
Carl Christian Liebe \\ Department of Electrophysics \\ Building 322 \\ Technical University of Denmark \\ DK - 2800 Lyngby \\ Denmark
}

\section{ABSTRACT}

A software system for a star imager for "on-line" satellite attitude determination is described. The system works with a single standard commercial CCD-camera with a high aperture lens and an on-board star catalogue. It is capable of both an initial coarse attitude determination without any a priori knowledge of the satellite orientation and a high accuracy attitude determination based on prediction and averaging of several identified star constellations. In the high accuracy mode the star imager aims at an accuracy better than 2 are sec. with a processing time of less than a few seconds. The star imager is developed for the Danish "micro satellite" Oersted.

\section{KEY WORDS}

Determination of orientation, Star Imager, Pattern recognition of star constellations.

\section{INTRODUCTION}

Almost all spacecrafts need to know their attitude. Several ways to determine the attitude relative to a reference object exist. Table 1 below shows reference sources suited for determining the attitude and their corresponding theoretical accuracies [1].

High precision equipment for satellites has stressed the demand for extremely accurate determination of the attitude. Therefore the latest attitude determination systems have relied on the most accurate reference, i.e. the stars. The principle in attitude determination by the stars is as follows: some image forming device (e.g. a CCD-camera) is directed toward the

Manuscript received June 9,1992 0885/8985/93 1993 IEEE
Table 1.

\begin{tabular}{ll}
\hline Reference object & Potential accuracy \\
Stars & 1 arcsecond \\
Sun & 1 arcminute \\
Earth (Horizon) & 6 arcminute \\
RF beacon & 1 arcminute \\
Magnetometer & 30 arcminute \\
\hline
\end{tabular}

stars. If the image can be matched to a reference, the direction of the imaging device is known, and thus the attitude of the spacecraft. One approach is to sweep the sky with a spectral sensitive device in order to recognize a known spectral distribution from a specific star. When the star is recognized the attitude control system locks on the specific star. Another approach has been a coarse determination of the attitude by others means (e.g., gyros), and then to fine tune the attitude by utilizing a CCD-camera image including a priori known constellation. Others have simply recorded the image from a CCD-camera together with the measured payload data, and then transmitted the lot to the earth for further automatic or manual processing. The disadvantages of these systems are high weight $(10-50 \mathrm{~kg})$, large power consumption and no possibility to determine the attitude without a coarse estimate of the attitude beforehand. These constraints are however incompatible with the future micro satellites, and therefore new methods of attitude determination must be developed.

The proposed Danish micro satellite "Oersted" is a micro satellite, whose purpose is to map the magnetic field of the earth. The main instrument is a vector magnetometer with an accuracy better than $2 \mathrm{nT}$. In order to utilize the extreme precision of the vector magnetometer the orientation must be determined with an accuracy better than $20 \mathrm{arc} \mathrm{sec}$. This implies that the attitude instrument (the star imager) must be placed very close to the magnetometer. 


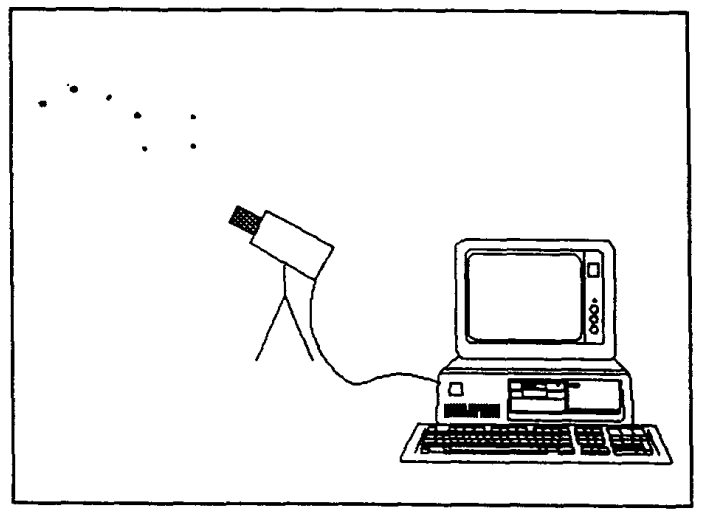

Fig. 1. The Star Imager, Principle of the System

The star imager must meet the following demands:

- weight of the attitude instrument less than $3 \mathrm{~kg}$;

- the power consumption below 8 Watts;

- ability to perform an attitude determination without a priori knowledge of the attitude;

- accuracy better than 20 arc. sec.; and

- a magnetic "clean" sensor.

A market survey showed that no available system met these specifications. Therefore a feasibility study was initiated to determine whether it is possible to construct a star imager within these limits. The study revealed the remarkable result that it is indeed possible to construct the star imager with an accuracy better than 2 arc sec.! This is even achieved by utilizing commercial available technology. The principle is as follows (Fig. 1). A CCD-camera is pointed towards the stars. The CCDimage is subjected to a number of corrections and compared to an internal star catalogue and thus identified. Based on the known star positions in the sky the attitude of the satellite can be determined. The system is based on state-of-the-art available consumer components and thus has the potential of being a true low cost system.

\section{STAR CATALOGUE AND DATABASE}

The high precession of the star imager stems from the very accurate knowledge of all the visible stars in the sky and the high reliability of $\mathrm{CCD}$-cameras. In order to differentiate between the single stars some characteristics to identify the stars in the sky are needed. To derive that the following two collections of star data are required:

- A star catalogue (SC); and

- A star database (SDB).

The star catalogue is a collection of data including the position of the stars. The star database is a collection of data containing position invariant characteristics of the stars. Problems related to the construction of the star database are: 1) how to find well suited features to characterize a star; and 2) how to include the uncertainty on the measured data in the search algorithms.

\section{Construction of the Star Catalogue}

The star catalogue includes the declination, right ascension, and magnitude of the stars as detected by the CCD-camera. It includes the 1539 brightest stars, and it is based on the PPM catalogue [4]. It is compiled with the following corrections of the raw star data:

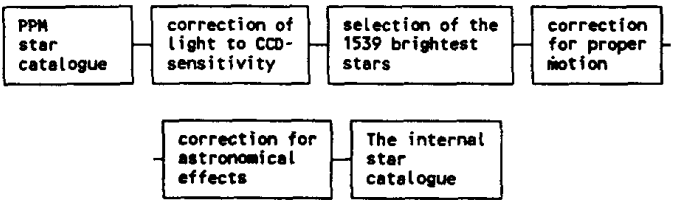

PPM star catalogue.

The PPM catalogue [4] was selected for the construction of the star catalogue. It is one of the latest and most accurate star catalogues available. The PPM catalogue includes information about the position of the stars, proper motion, magnitude, spectral class, uncertainty etc.

Correction to $C C D$-sensitivity.

The light intensities of the PPM catalogue are in the "V" magnitude system (or can easily be converted to that system). The CCD-chip has a spectral sensitivity different from the " $V$ " system, and corrections. must be made accordingly. The "V" magnitude is based on the wavelengths in the interval $480 \mathrm{~nm}$ to $650 \mathrm{~nm}$, which almost corresponds to the sensitivity of the human eye. If a star is considered a "blackbody-radiator" and furthermore neglecting that some wavelengths are absorbed in the chromosphere of the stars, the CCD brightness can be calculated:

$\langle C C D$ brigthness $\rangle=\left\langle\right.$ Visual brigthness $>\cdot C \frac{\sum_{\lambda=0}^{-} M_{e \lambda} \cdot S_{\lambda} \cdot T_{\lambda}}{\sum_{\lambda=0}^{\infty} M_{e \lambda} \cdot V_{\lambda}}$

where $\mathrm{M}_{\mathrm{e} \lambda}=$ spectral radiation, $\mathrm{V} \lambda=$ eye spectral sensitivity, $\mathrm{S} \lambda=\mathrm{CCD}$ spectral sensitivity, $\mathrm{T} \lambda=$ transmission coefficient of the CCD-lens and $\mathrm{C}$ is a camera amplifier constant.

Additionally the reflection in the lens depends on the angle of incidence. The stars closest to the rim of the picture is reflected the most (i.e., transmitted less). Therefore they will appear weaker than stars in the center of the picture. This phenomenon must be corrected for.

Correction for proper motion.

In the PPM catalogue proper motion and position of the stars are given to epoch 2000. Consequently it is straightforward to calculate the position to a certain time. The star catalogue is constructed to the end of year 1994 (based on the launch). 
The contribution from the precession is irrelevant due to the fact that the satellite is not making earth observations.

Nutation

As in the case with precession correction is irrelevant due to space operation.

Aberration

Aberration can be divided into contributions from 3 velocities: 1) the solar system velocity; 2) the earth velocity; and 3 ) the satellites own velocity.

1. The star catalogue is already in heliocentric coordinates.

2. The average velocity of the earth is $29.79 \mathrm{~km} /$ sec with a maximum correction on 20.48 arc sec.

3. The "Oersted" orbit is a circular polar orbit at $600 \mathrm{~km}$ height, which implies a tangential velocity of $8,3 \mathrm{~km} / \mathrm{sec}$. This gives a correction up to 5.7 arc sec.

Parallax

There has not been any corrections for parallax due to the following: 1) the effect only appears on the very few closest stars and with maximum on $0.85 \mathrm{arc} \mathrm{sec}$; and 2 ) the determination of the attitude is based on all the stars in the picture. Accordingly parallax error on a single or two stars will not affect the result significant.

\section{Relativistic light bending}

The CCD-chip is not constructed to point near the sun. Hence stars close to the sun are not registered. Also the maximum deviation of a star position is 0.1 arc sec. due to the relativistic light bending. Therefore the effect is not included.

\section{Star Characteristics}

In order to recognize stars in a CCD-image, some characteristics to distinguish between stars must be determined. Since the CCD-image only covers a small part of the firmament (in the present case $30^{\circ}$ ), it is impossible to search for wellknown star constellations like Orion, Big Dipper, etc. Hence more local characteristics of each star must be utilized. Among these are:

- angular distances to the closest neighbouring stars;

- spherical angles between the closest stars; and

- the magnitude.

Fig. 2 shows the distribution of the number of stars in the camera field of view, when the orientation of the optical axis is selected at random. An opening angle on $30^{\circ}$ was selected, as a trade off between the database size and the quality of the lens.

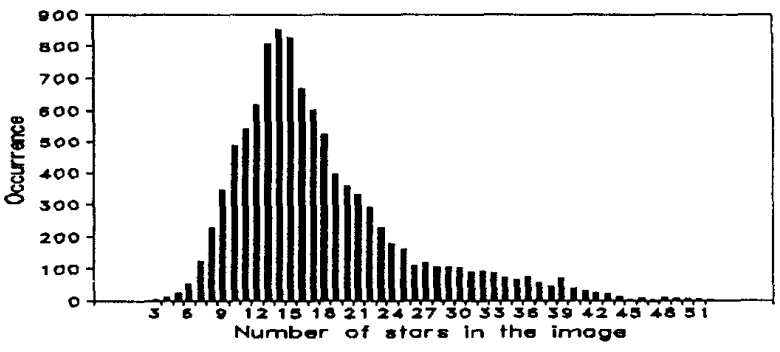

Fig. 2. Number of Stars in the Image. Opening angle $30^{\circ}$. The CCD-camera is Registering Stars Brighter than Optical Class 41/2. The Average Number of Stars is 18.9 (10.000 Simulations).

One obvious feature by which to characterize a star and its nearest surroundings is the angular distances to the closest neighbours. Figures 3 and 4 show the distribution of angular distance for the first and second neighbouring star (all brighter than optical class $4 \frac{1}{2} \approx 1107$ stars).

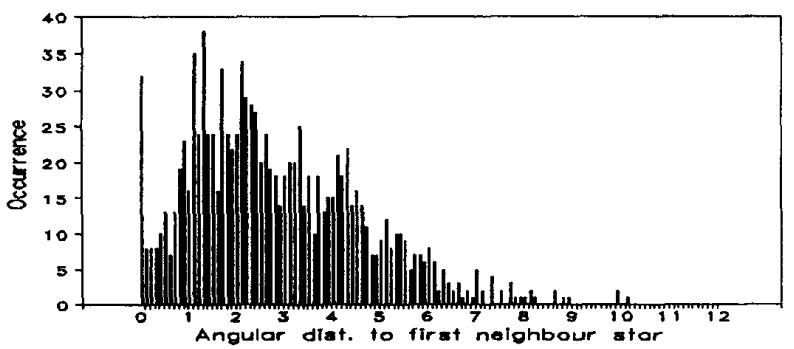

Fig. 3. Distribution of Distances (in degrees) to First Neighbouring Star Based on the 1107 Brightest Stars, the Peak at 0.0 - 0.1 is due to Double Star Systems

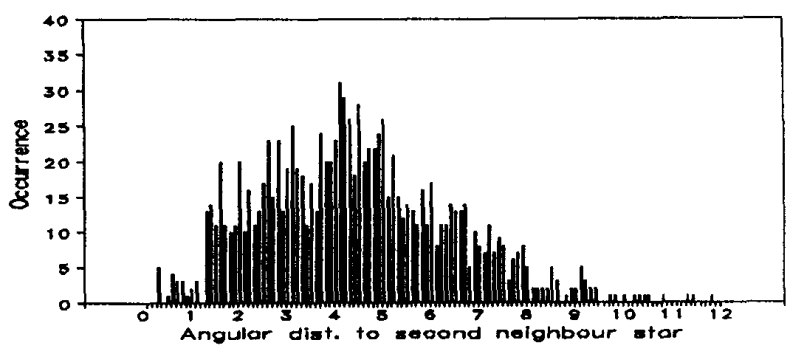

Fig. 4. Distribution of Distance (in degrees) to Second Neighbouring Star for the 1107 Brightest Stars 
Another major characteristic is the angle between the closest neighbouring stars (angle in the spherical triangle: first neighbouring star - star - second neighbouring star). As presumed the dispersion of angles between two neighbouring stars is nearly uncorrelated as Figure 5 shows. A flat distribution would be expected for homogenous distributed stars.

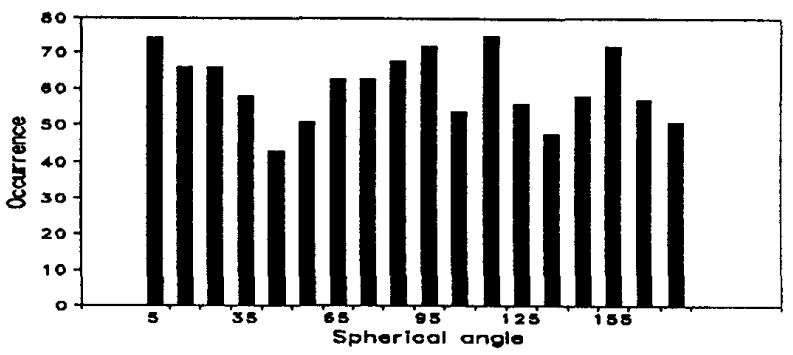

Fig. 5. Angle Between First and Second Neighbouring Star Based on the 1107 Brightest Stars

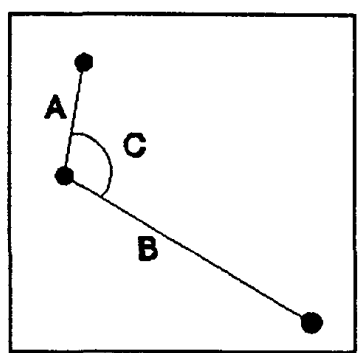

Fig. 6. The Triangular Feature. A: The Angular Distance to the First Neighbouring Star; B: the Angular Distance to Second Neighbouring Star; C: the Angle Between 1. and 2. Neighbouring Stars

Another obvious feature by which to characterize a star is its magnitude. However brightness as such is not applicable in the present case as noise limits the precision with increasing uncertainty for fainter stars. This implies that the distance to the first and second neighbour and the angle between them have been chosen as the characterizing parameters for the database (Figure 6). A subconstellation with these features is named a star triplet and its Parameter values the Triangular Feature (TF).

\section{Including Uncertainty in the Database}

In the CCD-image the positions and the magnitudes of the stars have an associated uncertainty. Therefore the characteristics of a measured CCD-star are not identical to the original star in the database (Figures 7 and 8). It is important to develop a search routine that will operate in spite of these uncertainties. This is referred to as an "open-end" structure of a database, which implies that the search algorithms will operate even when some of the stars occasionally fall below the detection limit.

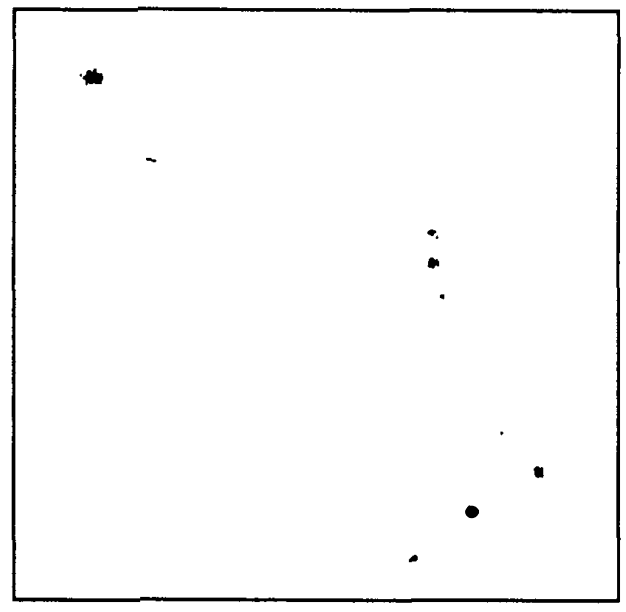

Fig. 7. A Primarily CCD-Image Containing a Part of Orion and Rigel. The star Not Included in the Star Catalogue is the Orion Nebula (M42).

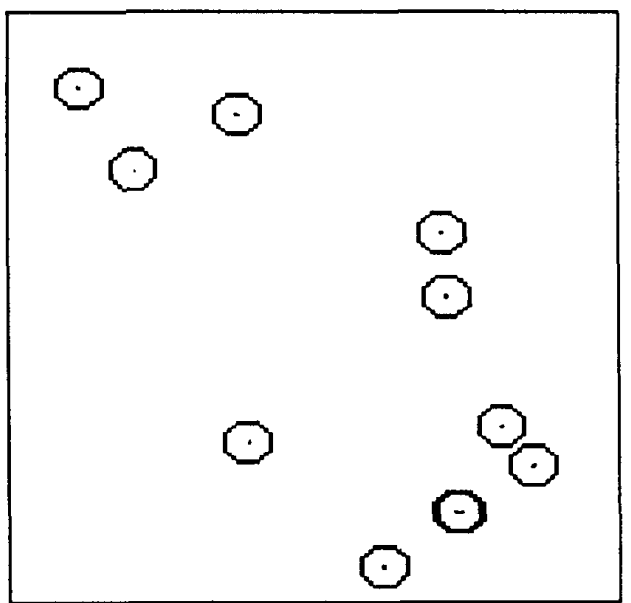

Fig. 8. The Contents of the Star Catalogue. The Stars Not Present in the CCD-Picture are Due to the Uncertainty on the Magnitude. The M42 in the Opposite Picture is Not Included in the Database and is Dealt with as a "False" Star (Described Later)

\section{Inclusion of Position-Uncertainty in the Database}

The CCD star images delivers (by utilizing the hyperaccuracy technique) a star position with a maximum deviation of 3 arc $\min \left(0.05^{\circ}\right)$ from its true position. To identify a specific star the TF is utilized (as described above). When a single star has a maximum absolute deviation on 3 arc min. the maximum deviation of the distance between two stars (relative) is 6 arc min. $\left(0.1^{\circ}\right)$. Furthermore data must be quanticed to minimize the memory requirement and processor loads. A reasonable choice is to quantice the distance in multiples of $6 \mathrm{arc}$ min. 
$\left(0.1^{\circ}\right)$ which corresponds to the relative uncertainty. As an example the angular distance $7.65^{\circ}$ will be transformed into class number 75,76 and 77 . The uncertainty on the angle between the first and second neighbouring star depends on the angular distances. It was decided to quantice the intervals of the angles to $5^{\circ}$. Consequently this will be registered in the same way as the distance.

As an example the TF identifier for a star could be the following:

distance to first neighbour:

distance to second neighbour:

angle between 1 . and 2 . neighbouring star:

$164.204^{\circ}$

This is quanticed into:

distance to first neighbour:

distance to second neighbour:

$55(5.596 \operatorname{div} 0.1)$

angle between 1 . and 2 . neighbouring star:

This is easier written as $[55,81,32]$.

As explained earlier the uncertainty must be included in the database. The uncertainty of the angle is calculated to $+1-$ $1.724^{\circ}$. The angle is therefore in the interval [ $164.204-1.724$; $164.204+1.724$ ] which is transformed into class 32 and 33 .

Just to cope with the uncertainty, this specific star must have the following entries in the database.

$$
\begin{array}{lllll}
54 & , & 80 & , & 32 \\
54 & , & 80 & , & 33 \\
54 & , & 81 & , & 32 \\
54 & , & 81 & , & 33 \\
54 & , & 82 & , & 32 \\
54 & , & 82 & , & 33 \\
55 & , & 80 & , & 32 \\
55 & , & 80 & , & 33 \\
55 & , & 81 & , & 32 \\
55 & , & 81 & , & 33 \\
55 & , & 82 & , & 32 \\
55 & , & 82 & , & 33 \\
56 & , & 80 & , & 32 \\
56 & , & 80 & , & 33 \\
56 & , & 81 & , & 32 \\
56 & , & 81 & , & 33 \\
56 & , & 82 & , & 32 \\
56 & , & 82 & , & 33
\end{array}
$$

Inclusion of Magnitude-Uncertainty in the Database

A star is only detected if the magnitude is above a given threshold level compared to the noise. Due to the uncertainty of the magnitude one cannot determine whether a star is above or below the threshold and hence if it will be registered. The uncertainty of the stellar magnitude is being included in the following way: Among the 1100 brightest stars there is a empirical determined uncertainty of $25 \%$. The 1100 brightest stars correspond to a specific brightness threshold value. If a star is below $75 \%$ of this limit, one can be sure that the star will not be detected. If the star is between $75 \%$ and $125 \%$ of the detection limit it might be detected (in this category double stars are included). If a star is above the $125 \%$ limit it will positively be detected. Hence there are two categories of stars which have to be included in the SDB namely the "certain" and the "maybe" stars. Registration of the nearest stars requires that all combinations are included.

This is illustrated in the following example.

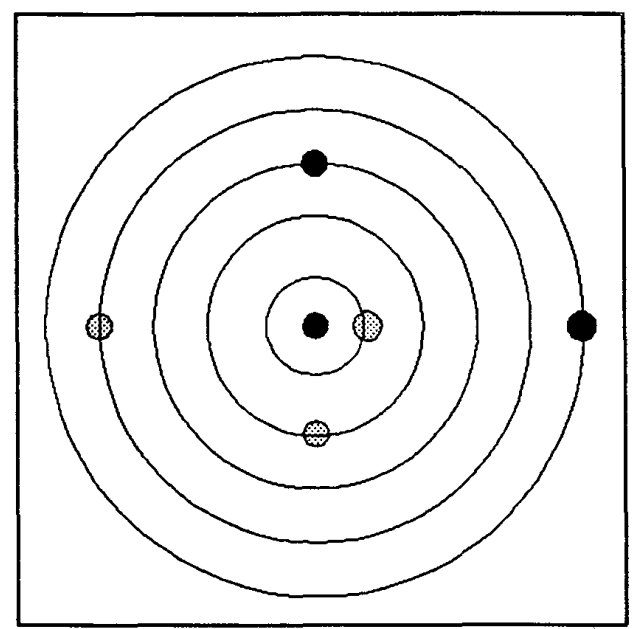

Fig. 9. The Star Which is to be Registered (in the center). A Neighbouring Star of the Category "Maybe" is Symbolized as $\bigcirc$. A Neighbouring Star of the Category "Certain" is Symbolized as

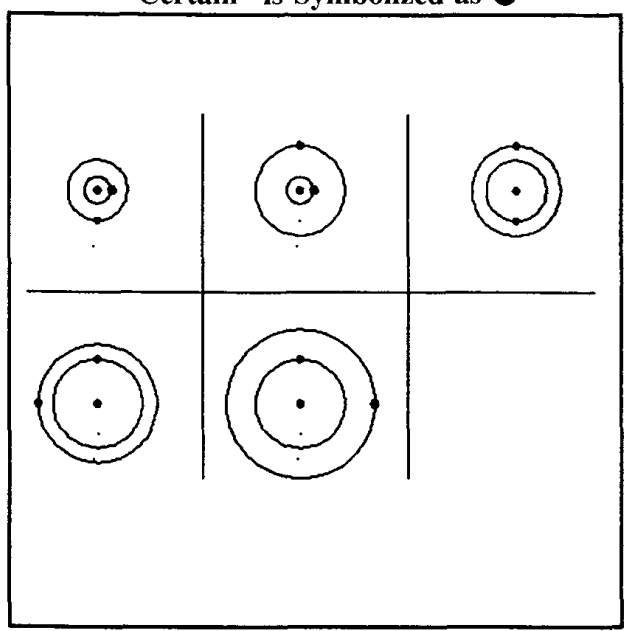

Fig. 10. The Star has to be Registered in the Depicted Constellations

The following shows a typical star from the implemented database at all its neighbouring constellations (NBC).

Each of these NBC's is affected by the uncertainty of the position (described in previous section). Hence entries in the SDB have to be made accordingly. Figure 11 shows all the entries in the database needed to register this specific star.

It may seem odd to include this amount of entries, but attention is called to the fact that the alternative is to operate with the raw SC. This would place a heavy burden on the onboard CPU. 
Distance to first Distance to second Angle between $1 \& 2$ neighbouring star $\left({ }^{\circ}\right)$ neighbouring star $\left(^{\circ}\right)$ neighbouring $\operatorname{star}\left({ }^{\circ}\right)$

$\begin{array}{lrr}5.596, & 8.191, & 164.204 \\ 5.596, & 8.329, & 30.680 \\ 5.596, & 10.102, & 7.542 \\ 5,596, & 12.306, & 58.521 \\ 5,596, & 12.754, & 13.597 \\ 8,191, & 8.329, & 165.117 \\ 8,191, & 10.102, & 156.662 \\ 8,191, & 12.306, & 137.275 \\ 8,191, & 12.754, & 150.607 \\ 8,329, & 10.102, & 38.222 \\ 8,329, & 12.306, & 27.841 \\ 8,329, & 12.754, & 44.276\end{array}$

\begin{tabular}{|c|c|c|c|c|}
\hline $\begin{array}{l}54,80,32 \\
54,80,33 \\
54,81,32 \\
54,81,33 \\
54,82,5 \\
54,82,6 \\
54,82,32 \\
54,82,33 \\
54,83,5 \\
54,83,6 \\
54,84,5 \\
54,84,6 \\
54,100,0 \\
54,100,1 \\
54,100,2 \\
54,101,0 \\
54,101,1 \\
54,101,2 \\
54,102,0 \\
54,102,1 \\
54,102,2 \\
54,122,11 \\
54,122,12 \\
54,123,11 \\
54,123,12 \\
54,124,11 \\
54,124,12 \\
54,126,2 \\
54,126,3 \\
54,127,2 \\
54,127,3 \\
54,128,2 \\
54,128,3 \\
55,80,32 \\
55,80,33 \\
55,81,32 \\
55,81,33 \\
55,82,5 \\
55,82,6 \\
55,82,32 \\
55,82,33 \\
55,83,5 \\
55,83,6 \\
55,84,5 \\
55,84,6\end{array}$ & $\begin{array}{l}55,100,0 \\
55,100,1 \\
55,100,2 \\
55,101,0 \\
55,101,1 \\
55,, 101,2 \\
55,102,0 \\
55,102,1 \\
55,102,2 \\
55,122,11 \\
55,122,12 \\
55,123,11 \\
555,123,12 \\
55,124,11 \\
55,124,12 \\
55,126,2 \\
55,126,3 \\
55,127,2 \\
55,127,3 \\
55,128,2 \\
55,128,3 \\
56,80,32 \\
56,80,33 \\
56,81,32 \\
56,81,33 \\
56,82,5 \\
56,82,6 \\
56,82,32 \\
56,82,33 \\
56,83,5 \\
56,83,6 \\
56,84,5 \\
56,84,6 \\
56,100,0 \\
56,100,1 \\
56,100,2 \\
56,, 101,0 \\
56,101,1 \\
56,101,2 \\
56,102,0 \\
56,102,1 \\
56,102,2 \\
56,122,11\end{array}$ & $\begin{array}{l}56,123,12 \\
56,124,11 \\
56,124,12 \\
56,126,2 \\
56,126,3 \\
56,127,2 \\
56,127,3 \\
56,128,2 \\
56,128,3 \\
80,82,32 \\
80,, 82,33 \\
80,83,32 \\
80,83,33 \\
80,84,32 \\
80,84,33 \\
80,100,30 \\
80,100,31 \\
80,101,30 \\
80,101,31 \\
80,102,30 \\
80,102,31 \\
80,122,26 \\
80,122,27 \\
80,123,26 \\
80,123,27 \\
80,124,26 \\
80,124,27 \\
80,126,29 \\
80,126,30 \\
80,127,29 \\
80,127,30 \\
80,128,29 \\
80,128,30 \\
81,82,32 \\
81,82,33 \\
81,83,32 \\
81,83,33 \\
81,84,32 \\
81,84,33 \\
81,100,30 \\
81,100,31 \\
81,101,30 \\
81,101,31 \\
811,102,30 \\
81,102,31\end{array}$ & $\begin{array}{l}81,122,26 \\
81,122,27 \\
81,123,26 \\
81,123,27 \\
81,124,26 \\
81,124,27 \\
81,126,29 \\
81,126,30 \\
81,127,29 \\
81,127,30 \\
81,128,29 \\
81,128,30 \\
82,32,32 \\
82,82,33 \\
82,83,32 \\
82,83,33 \\
82,84,32 \\
82,84,33 \\
82,100,7 \\
82,100,8 \\
82,100,30 \\
82,100,31 \\
82,101,7 \\
82,101,8 \\
82,101,30 \\
82,101,31 \\
82,102,7 \\
82,102,8 \\
82,102,30 \\
82,102,31 \\
82,122,5 \\
82,122,6 \\
82,122,26 \\
82,122,27 \\
82,123,5 \\
82,123,6 \\
82,123,26 \\
82,123,27 \\
82,124,5 \\
82,124,6 \\
82,124,26 \\
82,124,27 \\
82,126,8 \\
82,126,9 \\
82,126,29\end{array}$ & $\begin{array}{l}82,126,30 \\
82,127,8 \\
82,127,9 \\
82,127,29 \\
82,127,30 \\
82,128,8 \\
82,128,9 \\
82,128,29 \\
82,128,30 \\
83,100,7 \\
83,100,8 \\
83,101,7 \\
83,101,8 \\
83,102,7 \\
83,102,8 \\
83,122,5 \\
83,122,6 \\
83,123,5 \\
83,123,6 \\
83,124,5 \\
83,124,6 \\
83,126,8 \\
83,126,9 \\
83,127,8 \\
83,127,9 \\
83,128,8 \\
83,128,9 \\
84,100,7 \\
84,100,8 \\
84,101,7 \\
84,101,8 \\
84,102,7 \\
84,102,8 \\
84,122,5 \\
84,122,6 \\
84,123,5 \\
84,123,6 \\
84,124,5 \\
84,124,6 \\
84,126,8 \\
84,126,9 \\
84,127,8 \\
84,127,9 \\
84,128,8 \\
84,128,9\end{array}$ \\
\hline
\end{tabular}

Fig. 11. References in Data Base for a Typical Star. Average in the Data Base is 180 References

\section{Star Exchange Due to Uncertainty on the Position}

Due to the uncertainty a possible star exchange has to be considered as depicted in Figures 12 and 13.

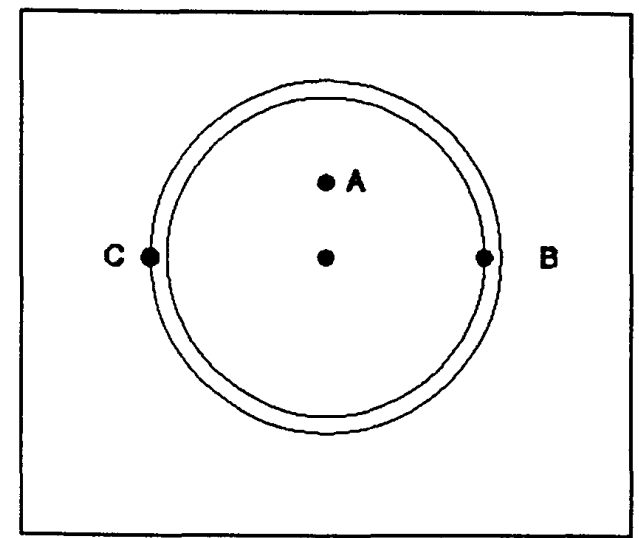

Fig. 12. Star with its 3 Neighbouring Stars

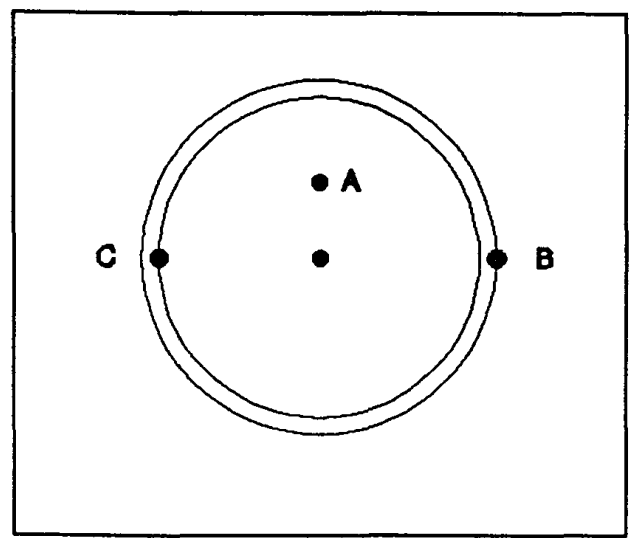

Fig. 13. Star with its 3 Neighbouring Stars and Uncertainty on Their Position

Figure 12 shows a star with its three neighbouring stars which are all in category "certain." Normally the star C will not be used for identification. However, the uncertainty on the angular distance to $\mathrm{B}$ and $\mathrm{C}$ is inside their respective uncertainties, and therefore the situation can be as shown in Figure 13, where star $\mathrm{C}$ is interpreted as the second nearest neighbour. This possible position exchange occurs only when the exchanged stars are very close to the first or second neighbouring star. Hence it is necessary to include them in the NBC entries. If worst-case is included all stars within 4 times the intervals of uncertainty have to be registered.

\section{ALGORITHMS}

The objective is to construct a "star-imager" able to perform both a coarse general attitude determination and subsequently a very accurate attitude determination. To reduce the weight and cost of the system, it is based on only one camera and lens. It is convenient to separate the software in two operating modes:

1. Initial estimate (IE) is used when no a priori knowledge of the camera orientation exists. 
2. Predictional estimate (PE) is a estimate where the coarse attitude is known. The purpose of the PE routine is to calculate the optical axis with greatest possible accuracy.

The IE routine is typically applied in the tumbling fase, before earth control has established contact (after the satellite is injected into orbit). When several images have been identified through the IE routine, the rotation of the satellite can be calculated, and the pointing direction of the camera can be calculated as a function of time. At this point the system switches to the PE mode in which a fine-tuning of the calculated attitude is performed.

\section{Initial Estimate}

The IE algorithm uses the TF to identify the stars in the image. As a first step all stars are discarded, when the distance to the second nearest neighbour is larger than the star's distance to the rim of the picture (to make sure that the nearest neighbours in fact are in the field of view). The remaining star triplets are searched for in the database. This search will result in a few star candidates fitting the same characteristics. The right candidate is easily selected, as the distances between the right candidates are known. Then the only remaining task is to calculate the optical axis based on the identified and verified stars. The described procedure ensures a necessary systemtolerance in situations where extra objects are in the field of view (e.g., planets or satellites) or when a bright star for some reason should disappear from the view, situations which indeed can occur.

Furthermore it cannot be included if a star is outside its uncertainty limits. All this is error situations which result in either the nearest stars are missing or false artificial nearest neighbours appear. This results in a wrong identification by

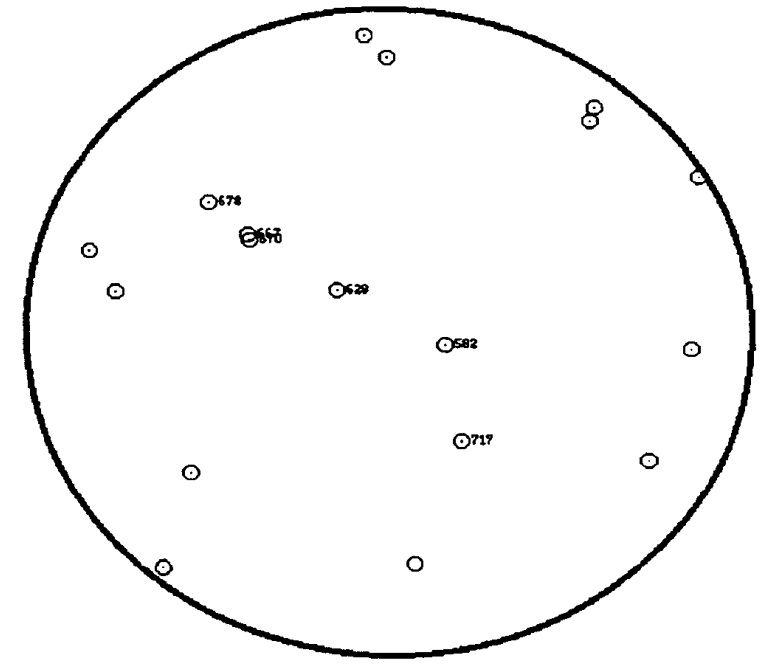

Fig. 14. Image from the IE Routine. Image to be Recognized Includes the Big Dipper. There is a circle around every star. Numbers at stars refer to the internal star catalogue. If no number is stated the star is too close to the rim of the picture the star database. A "false" or missing star will then not only be a source of error to its own detection, but will possibly lead to misidentification of the surrounding stars. The only way to reduce this problem is to ensure to have many stars in the field of view. For this reason a relatively high opening angle of the image should be chosen. Hereby a high immunity against errors of this character will be obtained. The table below summarizes a statistical analysis of different error situations which might occur along with the success rate of the IE routine.

Table 2. Different Error Situations and the Associated Possibility for a Correct IE. The Probabilities are Calculated through 500 Simulations with Each Type of Error

Situation

Probability for Correct Guess

All stars included and inside tolerance limits

$94.6 \%$

1 false star

$85.2 \%$

1 star outside the tolerance limits

$85.2 \%$

2 stars outside the tolerance limits

$78.6 \%$

1 star outside the tolerance limits and

$72.0 \%$

1 false star

1 star missing

$88.4 \%$

\section{Predictional Estimate}

The PE routine uses the coarse a priori determined attitude to create an image with the expected constellation based on the star catalogue. The stars in this image are matched to the stars in the camera image. This is done by least distance fit between any two stars from the two images. Distance deviations between the corresponding stars are calculated and subsequently minimized by differential variations of the presumed camera rotation and direction. The method is illustrated graphically in Figures 15, 16 and 17.

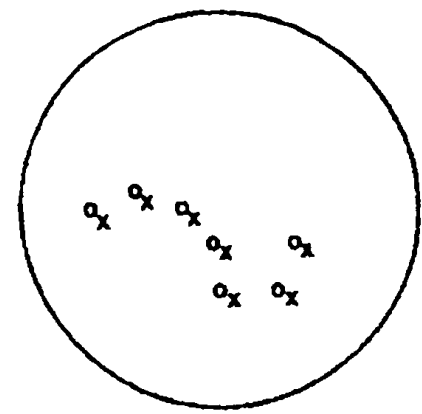

Fig. 15. The CCD-Star Constellation (x) and the Expected Constellation (o)

The major result in this study is the obtainable accuracy. The accuracy of the optical axis determination depends on the accuracy of the CCD-measurement and the number of stars in the image

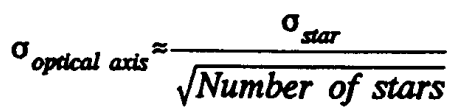




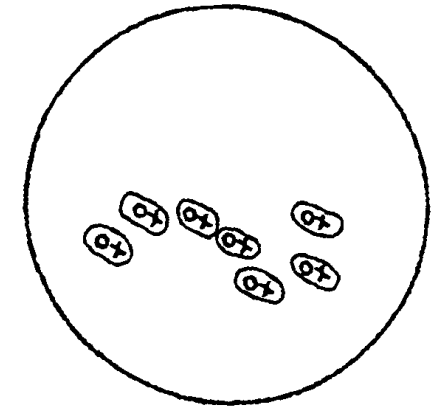

Fig. 16. The Corresponding Stars

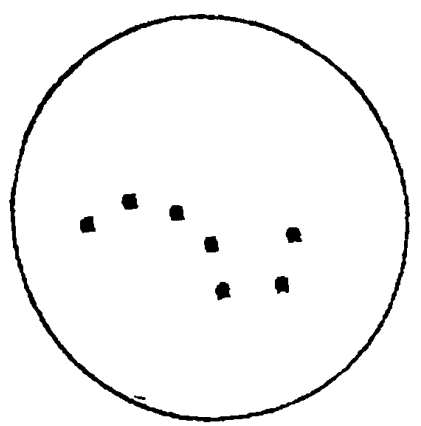

Fig. 17. Adjusted Guess

where star is the position accuracy of a single star in a CCDimage and optical axis is the accuracy of the calculated axis. A test was performed with 10,000 random PEs. The accuracy of the estimate was registered as a function of number of stars in the field of view (Figure 18).

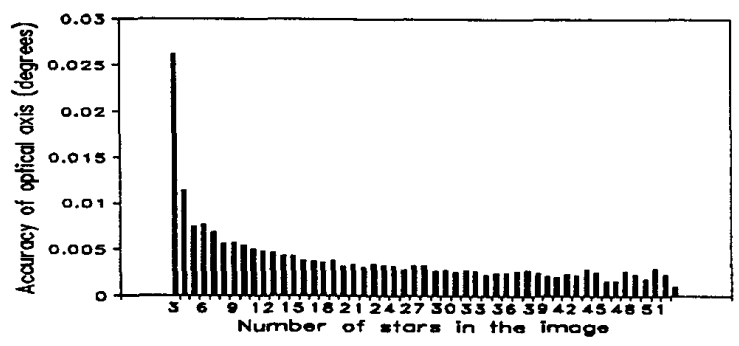

Fig. 18. Accuracy of Optical Axis as a Function of Number of Stars in Field of View. The Average is 14.6 Arc Sec.

It is evident that not all images are perfect (as an example a planet might appear in the field of view). Table 3 shows the accuracies obtained in a number of different error simulations.

The accuracy can be considerably increased by calculating accululated averages of several successive predictional guesses.

The key numbers of the star imager and its attitude determination software are shown below in Table 4.
Table 3. The Accuracy of the PE

in Different Error Situations.

The Accuracies are Based on 500 Simulations of Each Error Situation

\begin{tabular}{lc}
\hline \multicolumn{1}{c}{ Situation } & Accuracy (Arc sec.) \\
All stars included and inside the tolerance & 14,6 \\
$\quad$ limits & \\
1 false star & 15,4 \\
1 star outside the tolerance limits & 14,7 \\
2 stars outside the tolerance limits & 16,7 \\
1 star outside the tolerance limits and & 19,4 \\
1 false star & \\
1 star missing & 14,6
\end{tabular}

\section{CONCLUSIONS}

The angular distance to the first and second neighbouring star and the angle between them give an almost unique identification. It is established that the software must operate in two modes (with and without a priori knowledge of the attitude). The analysis shows that the developed method is able to determine the attitude of a satellite with an accuracy better than 15 arc sec. By accumulated averages accuracies better than 2 arc sec. can be obtained. The method proves itself as accurate, cheap, light-weight and with a low power requirement.

Table 4. Key Numbers

\begin{tabular}{lr}
\hline \multicolumn{1}{c}{ Subject } & \multicolumn{1}{c}{ Result } \\
\hline CCD-chip & NXA 1011 \\
Exposure time & $0.8 \mathrm{sec}$. \\
Pixels & $576 \times 604$ \\
Lens, opening angle & $30^{\circ}$ \\
Lens, aperture & $\mathrm{F}: 0.8$ \\
Accuracy, single star on & $1 / 5$ pixel, using \\
CCD-chip & 8000 \\
Number of stars registered & hyperaccuracy technique \\
with CCD & $(1100$ brightest are used $)$ \\
Success rate of IE routine & $95 \%$ in average situation
\end{tabular}

Typical time in IE-mode before $75-90 \%$ in abnormal situation entering PE-mode

IE, process time

$10 \mathrm{sec}$.

IE, accuracy, average

$P E$, success rate

PE, process time

PE, accuracy, average

Power consumption

Weight

Obtainable accuracy, accumulated averages

Database size (SDB and SC)

Number of entries in database
$2 \mathrm{sec}, 386 / 387$ processor board, $33 \mathrm{MHZ}$.

$0.1^{\circ}$ $100 \%$ $1 / 2 \mathrm{sec}$.

15 arc. sec.

$<8$ Watts

$=3 \mathrm{Kg}$, depending on the baffle construction

1 arc sec.

1 Mbyte

185.000 


\section{ACKNOWLEDGEMENT}

The author is grateful to Associate Professor John L. Jørgensen and Docent, Associate Professor Anders Damkjær for all the valuable suggestions and comments during the course of this work.

\section{REFERENCES}

[1] Peter Fortescue, John Stark: Spacecraft System Engineering, John Wiley \& Sons Ltd., Sussex, England.

[2] C. Elstner, G. Lichtenauer and W. Skarus: "ASTRO IM - ein neues Meßsystem für die Astroorientierung von Raumflugkörpern

Jenaer Rundschau 3 (1990).

[3] J. Curtis:

Attitude Monitor for the Jet-X-Ray Telescope.

Rutherford Appleton Laboratory, Private communications.

[4] S. Roeser, U. Bastion:

The PPM Catalog.

Astronomisches Rechen-Institut, Heidelberg, June 1989

[5] J.L. Jørgensen, A. Damkjær, C.C. Liebe: A project proposal for a star imager for accurate on-line attitude determination.

Annual meeting for Nordic Society for Space Research, 18-21 November 1991, Møn, Denmark.

[6] F. Primdal, P. Anker Jensen:

Dansk rumforskningsinstituts raket magnetometer ekspirenient. Gamma 56 (1984).

Carl Christian Liebe was born on October 16, 1966 in Copenhagen, Denmark. He received the M.S. degree in electrical engineering at the Technical University of Denmark in September 1991. He is currently working towards his Ph.D. degree in Pattern Recognition at the Department of Electrophysics, the Technical University of Denmark.

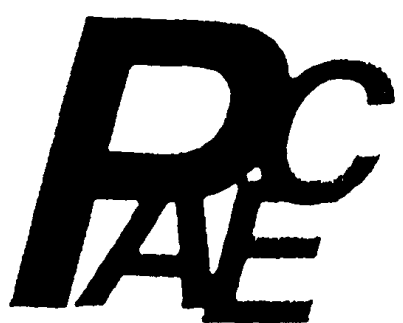

\section{IEEE Programs for Unemployed Members}

\author{
by
}

Paul J. Kostek, AESS PACE Chairman
The IEEE-USA Employment Assistance Committee has resource material and workshops that can be run by local Sections for unemployed members and interested employed members. The committee has developed a workshop and has a videotape on job hunting that Sections or Chapters can borrow. The videotape entitled, "How to Get a Better Job Quicker," can either be shown as part of a Section meeting or as part of a section developed workshop. You will need to work with your local Section PACE Chair, or AESS Chapter Chair to coordinate any activity.

The committee has created a workshop called "Job Seekers Workshop." Facilitator training was provided at the recent PACE Workshop. If you are interested in this workshop contact your Section's PACE Chair to see about holding a workshop in your area. A member of the committee or a facilitator who lives in your geographic area could be brought in to conduct the workshop

One of the most important ways to find a job is through personal networks. I always tell people that the IEEE should be a part of your network. By holding a Chapter or PACE meeting on employment topics, a Section helps it's members in developing and expanding their contacts. If you are an AESS Society Chapter Chair, I encourage you to contact your Section PACE Chair and discuss the possibilities for joint activities.

If you want more information, please feel free to contact Bill Anderson at the IEEE-USA office (202-785-0017) or contact me at 206-556-1102.

Pamphlet available: "The Defense Professional's Survival Guide for the 1990s," from the National Center for Career Change, (800)755-2411@\$2.95. 